\title{
Raymond Trousson, Denis Diderot ou le vrai Prométhée
}

Franco Piva

\section{(2) OpenEdition}

1 Journals

\section{Edizione digitale}

URL: https://journals.openedition.org/studifrancesi/26371

DOI: 10.4000/studifrancesi.26371

ISSN: 2421-5856

\section{Editore}

Rosenberg \& Sellier

\section{Edizione cartacea}

Data di pubblicazione: 1 avril 2007

Paginazione: 182-183

ISSN: 0039-2944

\section{Notizia bibliografica digitale}

Franco Piva, «Raymond Trousson, Denis Diderot ou le vrai Prométhée», Studi Francesi [Online], 151 (LI I I) | 2007, online dal 30 novembre 2015, consultato il 23 novembre 2021. URL: http:// journals.openedition.org/studifrancesi/26371 ; DOI: https://doi.org/10.4000/studifrancesi.26371

Questo documento è stato generato automaticamente il 23 novembre 2021.

\section{(c) $(1) \&$}

Studi Francesi è distribuita con Licenza Creative Commons Attribuzione - Non commerciale - Non opere derivate 4.0 Internazionale. 


\title{
Raymond Trousson, Denis Diderot ou le vrai Prométhée
}

\author{
Franco Piva
}

\section{NOTIZIA}

RAYMOND TROUSSON, Denis Diderot ou le vrai Prométhée, Paris, Tallandier, 2005, pp. 717.

1 Non è facile scrivere la storia di Diderot e della sua opera. A differenza di Rousseau, che si è consegnato, intus et in cute, nelle Confessions e nelle altre opere autobiografiche; a differenza di Voltaire, la cui vita può essere seguita e ricostruita praticamente giorno dopo giorno attraverso la sterminata corrispondenza e le numerose tracce della sua presenza lasciate nei diversi scenari all'interno dei quali si è trovato ad operare, Diderot ha parlato molto poco di sé. Come è stato giustamente fatto osservare, «L'écrivain le plus disert de sa génération [...] possédait aussi, la plume à la main, une étonnante aptitude à la discrétion sur lui-même». La corrispondenza rimastaci, pur preziosa per molti aspetti lascia ampie zone assolutamente scoperte: se si aggiunge che molte delle sue opere sono rimaste inedite, talvolta fino ad una data tutto sommato recente, e che della loro genesi egli ha detto spesso pochissimo, si comprende quanto sia difficile costruire una biografia sufficientemente precisa e fiable di un personaggio, come Diderot, che fino all'altro giorno era, peraltro, considerato poco più che il direttore dell'Encyclopédie. Non è un caso se la precedente grande biografia di Diderot, quella dell'americano Arthur Wilson, risale ad oltre trent'anni or sono. Lo sforzo di Raymond Trousson è, proprio per questo, ancora più meritorio ed apprezzabile. Vero è che egli ha potuto avvalersi di tutto quanto la ricerca su Diderot, molto vivace in questi ultimi decenni, è riuscita a mettere a disposizione degli studiosi sia per quanto riguarda una più precisa conoscenza di certi episodi della sua esistenza sia per quanto concerne una più esatta conoscenza di alcune delle sue opere e delle circostanze, spesso incerte e mal conosciute, che le hanno viste nascere, e che non sono affatto ininfluenti sul loro vero significato. La straordinaria familiarità che Trousson ha con l'autore di Jacques le fataliste, e con tutto il Settecento francese, oltre che con la letteratura critica che ha 
accompagnato Diderot dal secolo dei Lumi fino ai nostri giorni, ha consentito all'autore di questo bel libro di ricostruire la vita e l'opera dell'autore del Neveu de Rameau e della Religieuse in una maniera e con dei risultati capaci di soddisfare i palati più esigenti.

Il primo pregio del lavoro di Troussson riguarda il modo in cui è stata ricostruita la biografia di Diderot: inseguito, nella misura del possibile au jour le jour, tanto nella sua vita privata quanto nella sua vita di intellettuale e di scrittore multiforme. Da questa ricostruzione esce un personaggio almeno in parte nuovo, certamente più completo $\mathrm{e}$ complesso di quello che ci avevano presentato, anche per la scelta degli autori che avevano privilegiato l'uomo pubblico rispetto a quello privato, le biografie precedenti. La ricostruzione di Trousson consente di cogliere innanzitutto Diderot nei diversi aspetti della sua vita di uomo, di marito, di padre, di amico e di nemico, con le sue passioni i suoi entusiasmi, le sue debolezze, le sue idiosincrasie, di coglierlo soprattutto nelle sue più articolate ed a volte insospettabili contraddizioni. Contemporaneamente, e quasi dall'interno di questo complesso reticolo, assistiamo al progressivo formarsi del Diderot philosophe e della visione che, attraverso mille tentennamenti e cento aporie, Diderot va via via formandosi del mondo e dell'uomo, dalla sua straordinaria curiosità, non di rado favorita da circostanze fortunate (o fortuite?), che lo portano ad interessarsi degli argomenti più diversi e gli consentono di formarsi in pochi anni una cultura solo apparentemente disparate, in realtà tale da fornirgli i materiali per una riflessione via via più acuta ed approfondita, in grado di appréhender la realtà in tutta la sua complessità, fino alla vera e propria fascination che su Diderot esercita il libero gioco delle idee, anche di quelle apparentemente più strane e paradossali, che egli insegue con un'apparente nonchalance, in realtà con un'ostinata convinzione, lungo un percorso di cui si individua la traccia via via più precisa a mano a mano che si leggono le precise analisi che Trousson propone delle diverse opere che Diderot viene componendo, più che pubblicando; sicché seguendo il percorso proposto da Trousson il lettore ha la possibilità di seguire il complesso dipanarsi del pensiero del philosophe lungo le innumerevoli tappe attraverso le quali esso va via via snodandosi e costruendosi. Di questo pensiero le puntuali ricostruzioni e le analisi di Trousson consentono di cogliere, in maniera sempre più precisa a mano a mano che le pagine scorrono, le linee portanti, sia che si tratti di filosofia, sia che si tratti di teatro o di politica.

3 In questa ricostruzione il direttore dell'Encyclopédie ha certamente una parte importante: della grande impresa sono ripercorse tutte le tappe, da quelle più esaltanti a quelle più drammatiche, che l'autore ripercorre dal punto di vista di Diderot piuttosto che della loro realtà storica, in modo da far meglio cogliere al lettore le ricadute che esse hanno su di lui come uomo privato prima ancora che come uomo di cultura; ci pare tuttavia che più che sul responsabile della maggiore impresa editoriale, ed ideologica, dell'intero Settecento, l'accento, e lo sforzo di Trousson siano stati posti sull'altro Diderot, quello sul quale peraltro s'è incentrata l'attenzione della critica più recente, al fine di dare a colui nel quale la critica, dopo un lungo periodo di incertezza, di cui Trousson ha dato conto in un suo precedente libro (cfr. "Studi francesi», n. 149), ha individuato in maniera sempre più convinta il terzo componente della celebre triade illuministica quello spessore e quella dimensione profondamente originale che una lettura integrale, condotta su testi finalmente sicuri, ha reso non solo possibile in questi ultimi anni, ma a mano a mano anche sempre più sostanziale e convincente. 
4 Il lettore ha la possibilità di seguire passo passo questa ricostruzione e di apprezzarne le alte qualità anche grazie al particolare linguaggio usato da Trousson: un linguaggio criticamente ineccepibile, e purtuttavia sapido, ricco di immagini e di espressioni colorite che rivelano e rinviano a quel gusto per la lingua parlata che costituisce, come è noto, una delle cifre più cospicue della scrittura di Diderot: sicché il lettore ha, non di rado, l'impressione di trovarsi di fronte a Diderot, piuttosto che al suo biografo. Non c'è praticamente soluzione di continuità tra la lingua delle citazioni, giustamente molto frequenti, e la lingua di Trousson che anche da questo punto di vista ha saputo entrare appieno nel personaggio che ha inteso restituirci in tutta la sua umanità, oltre che nella complessità delle sue idee e nella ricchezza delle forme in cui queste idee Diderot ha cercato di trasmettere ai suoi contemporanei; senza riuscirci sempre come avrebbe voluto o sperato; in modi tuttavia per cui anche i tentativi non portati a termine, e giustamente valorizzati da Trousson, lasciano intravedere il senso e la direzione di un percorso che se non è sempre facile cogliere nelle sue complesse articolazioni, è però sempre affascinante e ricco di stimoli anche, se non soprattutto, per il lettore di oggi, che dalla ricostruzione di Trousson trarrà non solo gli elementi che faciliteranno il suo approccio ad una esistenza e ad un'opera indubbiamente complesse, ma anche le indicazioni che lo aiuteranno nella loro comprensione più autentica. 